\title{
Smart nanocarriers for drug delivery: controllable LSPR tuning
}

\author{
A.M. Lopatynskyi ${ }^{1}$, V.K. Lytvyn ${ }^{1}$, I.V. Mogylnyi ${ }^{1}$, O.E. Rachkov ${ }^{2}$, O.P. Soldatkin ${ }^{2}$ and V.I. Chegel $^{1}$ \\ ${ }^{1}$ V. Lashkaryov Institute of Semiconductor Physics, National Academy of Sciences of Ukraine \\ 41, prospect Nauky, 03680 Kyiv, Ukraine, \\ Phone: +38(044)525-56-26, e-mail: lop2000@ukr.net, lytvet@ukr.net,imogilnyu@gmail.com, \\ vche111@yahoo.com \\ ${ }^{2}$ Biomolecular Electronics Laboratory, Institute of Molecular Biology and Genetics \\ National Academy of Sciences of Ukraine, 150, Zabolotnyi str., 03143 Kyiv, Ukraine, \\ Phone: +38 (044) 200-03-28, e-mail: oleksandr_rachkov@yahoo.com,a_soldatkin@yahoo.com
}

\begin{abstract}
Gold nanostructures are considered as a potential platform for building smart nanocarriers that will form the basis of novel methods of targeted delivery and controlled release of drugs. However, to ensure maximum efficiency of gold nanoparticles upon the drug release via the plasmon-enhanced photothermal effect, it is necessary to optimize their spectral parameters for operation in the human body that requires both theoretical research and development of appropriate methods for nanostructures fabrication. In this work, mathematical modeling of light extinction spectral dependences for gold nanostructures of different morphology was performed to determine their geometric parameters that provide the occurrence of localized surface plasmon resonance (LSPR) in the red and near infrared regions of the spectrum, where the transparency window of biological tissues exists. Based on the results of previous studies and computer modeling, using hollow gold nanoshells to construct smart nanocarriers was found to be most reasonable. A protocol for production of these nanoparticles based on "silver-gold" galvanic replacement reaction, which is accompanied by a controlled shift of the LSPR wavelength position, was proposed and described in detail. It is shown that the loading of model biomolecules in hollow gold nanoshells significantly changes the output optical parameters of the system under investigation, which should be taken into account for matching with the laser excitation wavelength during the development of smart nanocarriers.
\end{abstract}

Keywords: localized surface plasmon resonance, smart nanocarriers, gold nanostructures.

Manuscript received 21.07.16; revised version received 06.09.16; accepted for publication 16.11.16; published online 05.12.16.

\section{Introduction}

The rapid development of nanobiotechnology can be a crucial step of mankind towards creation of novel methods and drugs for the treatment of various diseases $[1,2]$. New opportunities in medicine that will be available through advanced nanotechnology range from diagnostics of human body [3] to real means of practical therapy [4]. In particular, the creation of nanodrugs will promote a more specific therapy and provide local action, increasing treatment efficacy and reducing side effects. Therefore, the development of novel nanomaterials for applications in drug delivery systems is lately becoming a more and more popular field due to the unique optical, electronic, chemical and mechanical properties of these materials [4-7]. 
Metal nanoparticles can act as one of the key components of drug delivery systems; among them, the most attention of researchers was attracted to gold nanostructures of various shapes and sizes [8-10], which possess a number of significant advantages over other nanomaterials. In particular, they are chemically stable, biocompatible and can be functionalized with a large number of different nature ligands [11, 12]. Additionally, gold nanostructures support the excitation of localized surface plasmon resonance (LSPR) in the visible and near-infrared (NIR) spectral regions, which enables the use of local plasmon-enhanced photothermal effect (PPTE) [13, 14]. This phenomenon is due to gold nanostructures functioning as nanoscale lenses, focusing incident light in a confined localized area, which results in a conversion of high-density electromagnetic energy into heat that warms up nanostructures and their nearest environment. One of the possible applications of PPTE in metal nanoparticles have been successfully demonstrated in the development of new treatment modes for cancer, where heating of nanostructures by laser irradiation is used for the selective destruction of tumor cells [15-18]. In this direction, an important step was made by Elliott et al., who determined the quantitative characteristics of interaction with laser light for gold nanoshells with quartz cores to evaluate the impact of nanostructure concentration and laser power on PPTE [15]. Further, Stern et al. evaluated the effect of concentration of gold nanoshells with quartz cores in the treatment of prostate cancer in mice [16]. El-Sayed with colleagues used solid gold nanoparticles coated with antibodies against the EGF receptor for targeted delivery and photothermal treatment of epithelial carcinoma [17].

For the treatment that requires the use of chemical drugs or specific biopolymers (DNA sequences, immunoglobulines etc.), and not only direct heat influence, PPTE may be supplemented by the development of special "smart nanocarriers" (SN), which make possible the targeted delivery of drug/oligonucleotide/immunoglobuline molecules and their subsequent dosed release after controlled local irradiation of the desired area in the body [19]. In developing this type of $\mathrm{SN}$, one of the main tasks is to provide possibility for tuning the LSPR spectrum of gold nanostructures into the red and NIR regions, where light absorption by human tissues is minimal [20]. It is also important to take into account the changes in the LSPR spectrum induced in the process of drug loading into SN because of its dependence on the dielectric properties of the immediate environment [21]. In addition, the possibility of SN LSPR spectrum tuning is a prerequisite to achieve the greatest PPTE due to matching the LSPR wavelength with a wavelength provided by existing sources of laser radiation [22]. Therefore, potentially the most useful for creating SN based on PPTE are gold nanostructures such as nanoshells with the dielectric core [23], nanorods [24], nanocages [25] and nanocontainers [26] due to their capacity of LSPR regulation in a wide range, which is defined primarily by the geometrical parameters of these nanostructures.

In this paper, geometric parameters of gold nanostructures with different morphology are theoretically grounded, which are necessary for their use in SN based on PPTE operating in the red and NIR spectral regions. Based on the results of simulation and search for the synthesis methods of relevant nanostructures, a protocol for the synthesis of hollow gold nanoshells (HGN) is proposed and analyzed, which supports the controlled tuning of the LSPR spectral position. Based on the experimental evaluation of drug loading into HGN using model biomolecules, the importance of taking into account the presence of organic component of the system during matching with the wavelength of laser radiation is shown.

\section{Theoretical consideration}

To determine the geometric parameters of gold nanostructures that can support LSPR excitation in the red and NIR spectral regions and serve as a basis for building SN, theoretical modeling of light extinction spectra was conducted for three types of model nanostructures: solid spherical gold nanoparticles, gold nanoshells with quartz cores and HGN. Also, in the case of solid gold nanoparticles, the influence of existing dielectric coating, which simulates the organic components of SN (e.g., a polymer or biomolecular coating with loaded drugs), on the optimal size of nanostructures in terms of LSPR spectral position was considered.

Calculations of light extinction spectra for the abovementioned gold nanostructures were conducted using the previously developed approach based on Mie theory for two-component spherical particles consisting of a core and homogeneous shell [27], which was modified to consider gold nanoshells. Namely, the size effect of the electron mean free path reduction with a decrease in the size of the nanostructures was taken into account differently for solid gold nanoparticles and nanoshells. In particular, the effective electron relaxation time dependent on the size of nanostructures for solid gold nanoparticles was calculated according to the equation

$\tau_{\text {eff }}(R)=\left(\tau_{\text {bulk }}^{-1}+A \frac{V_{f}}{R}\right)^{-1}$,

and in the case of gold nanoshells using the following formula [28]:

$\tau_{\text {eff }}\left(r_{1} r_{2}\right)=\left(\tau_{\text {bulk }}^{-1}+A \frac{V_{f}}{l_{\text {eff }}\left(r_{1} r_{2}\right)}\right)^{-1}$,

where $\tau_{\text {bulk }}=9.3 \times 10^{-15} \mathrm{~s}$ [29] is the electron relaxation time for bulk gold, $V_{f}=1.4 \times 10^{6} \mathrm{~m} / \mathrm{s}$ [30] Fermi velocity for gold, $R$ - solid nanoparticle radius, 
$A$ - constant taken equal to 1 [31-33], $l_{\text {eff }}$-electron effective mean free path in the metal nanoshell according to the model proposed by Granqvist and Hunderi [28, 34]:

$l_{\text {eff }}\left(r_{1} r_{2}\right)=\frac{1}{2}\left[\left(r_{2}-r_{1}\right)\left(r_{2}^{2}-r_{1}^{2}\right)\right]^{1 / 3}$,

where $r_{1}$ and $r_{2}$ are internal and external radii of the gold nanoshells, respectively.

The purpose of this simulation was to obtain for the nanostructures described above the LSPR wavelengths close to 650 and $808 \mathrm{~nm}$, which correspond to those of standard laser light sources and thus can provide maximum PPTE when being used to create SN. For this aim, a search for nanostructure geometrical parameters variants was carried out with subsequent calculation of the light extinction spectrum and determination of the spectral position of its maximum corresponding to the occurrence of LSPR. The calculation of optical constants for gold nanostructures was performed in the framework of the approach presented earlier [27], taking into account the relations (1)-(3). The refractive index of the ambient medium in all the considered systems was 1.333 (equals to that of water). The refractive index of dielectric coating, which simulates the organic components of $\mathrm{SN}$ in the case of solid gold nanoparticles, was 1.46 [27]. The refractive index of the core in the case of gold nanoshells with quartz cores was 1.45 , and in the case of HGN it was equal to the refractive index of the ambient medium (1.333).

The geometrical parameters simulation results for three types of nanostructures that provide LSPR excitation at the wavelengths close to 650 and $808 \mathrm{~nm}$ are shown in Fig. 1 and in Table. It should be noted that for gold nanoshells LSPR spectral position is determined by two geometric parameters - the diameter of the core/cavity and the thickness of the shell, so this simulation was carried out for three fixed values of the core/cavity diameters $(20,50$ and $100 \mathrm{~nm})$. The obtained results indicate that, to achieve the abovementioned LSPR spectral positions, one should use quite large solid gold nanoparticles (about 150 and $210 \mathrm{~nm}$ in diameter for the LSPR at 650 and $808 \mathrm{~nm}$, respectively). It was also found out that the optimal geometrical parameters for gold nanoshells with quartz cores and HGN are pretty close, which is unexpected and can be explained by the strong LSPR position dependence on the thickness of the gold shell, which was observed during simulation, especially for the small diameter cores. Also, for the considered gold nanoshells with a core/cavity diameter of $100 \mathrm{~nm}$, the minimum limit value of the dipolar LSPR mode wavelength that can be achieved and a corresponding thickness of the gold shell were found. It is worth noting that a general trend of LSPR bandwidth increase and appearance of additional shortwavelength maxima in the light extinction spectra, which correspond to the quadrupolar LSPR modes, was evidenced with increasing the size of nanostructures. (a)

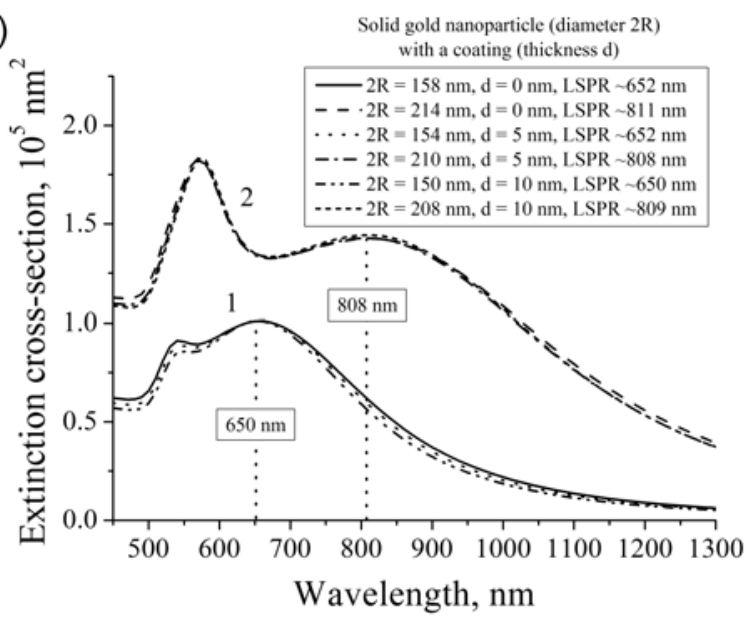

(b)

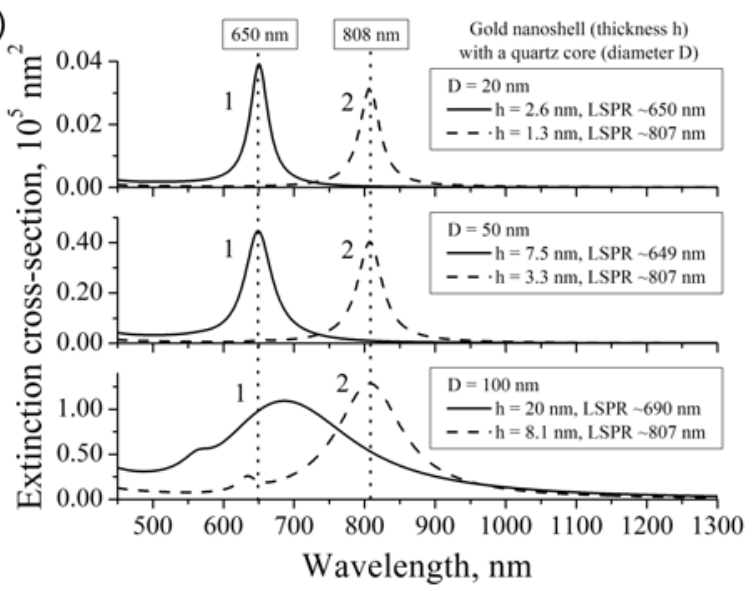

(c)

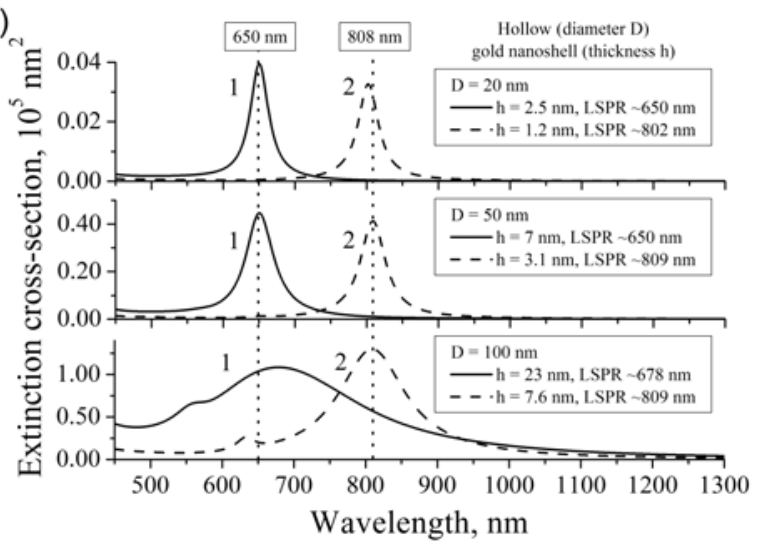

Fig. 1. Simulated light extinction spectra, which exhibit LSPR excitation at the wavelengths close to 650 (mark 1) and $808 \mathrm{~nm}$ (mark 2), for (a) solid gold nanoparticles with and without organic coating, (b) gold nanoshells with quartz cores and (c) HGN with different geometrical parameters, located in water. 
Table. Simulated geometrical parameters for gold nanostructures of different types, located in water, which provide LSPR excitation at the wavelengths close to 650 and $808 \mathrm{~nm}$.

\begin{tabular}{|c|c|c|}
\hline \multirow{2}{*}{ Nanostructure type } & $\begin{array}{c}\text { LSPR at } \sim 650 \\
\text { nm }\end{array}$ & $\begin{array}{l}\text { LSPR at } \\
\sim 808 \mathrm{~nm}\end{array}$ \\
\hline & \multicolumn{2}{|c|}{$\begin{array}{l}\text { Gold nanoparticle } \\
\text { diameter, } \mathbf{n m}\end{array}$} \\
\hline $\begin{array}{l}\begin{array}{l}\text { Solid gold nanoparticle } \\
\text { without coating }\end{array} \\
\end{array}$ & 158 & 214 \\
\hline $\begin{array}{l}\text { Solid gold nanoparticle with a } \\
\text { 5-nm-thick organic coating }\end{array}$ & 154 & 210 \\
\hline \multirow[t]{2}{*}{$\begin{array}{l}\text { Solid gold nanoparticle with a } \\
\text { 10-nm-thick organic coating }\end{array}$} & 150 & 208 \\
\hline & \multicolumn{2}{|c|}{ Gold shell thickness, nm } \\
\hline $\begin{array}{l}\text { Gold nanoshell with a quartz } \\
\text { core diameter of } 20 \mathrm{~nm}\end{array}$ & 2.6 & 1.3 \\
\hline \begin{tabular}{|l|} 
Gold nanoshell with a quartz \\
core diameter of $50 \mathrm{~nm}$
\end{tabular} & 7.5 & 3.3 \\
\hline $\begin{array}{l}\text { Gold nanoshell with a quartz } \\
\text { core diameter of } 100 \mathrm{~nm}\end{array}$ & $\begin{array}{c}\text { Not found }(20 \\
\mathrm{nm} \text { for LSPR at } \\
\sim 690 \mathrm{~nm})\end{array}$ & 8.1 \\
\hline $\begin{array}{l}\text { HGN with a cavity diameter of } \\
20 \mathrm{~nm}\end{array}$ & 2.5 & 1.2 \\
\hline $\begin{array}{l}\text { HGN with a cavity diameter of } \\
50 \mathrm{~nm}\end{array}$ & 7 & 3.1 \\
\hline $\begin{array}{l}\text { HGN with a cavity diameter of } \\
100 \mathrm{~nm}\end{array}$ & $\begin{array}{l}\text { Not found }(23 \\
\mathrm{nm} \text { for LSPR at } \\
\sim 678 \mathrm{~nm})\end{array}$ & 7.6 \\
\hline
\end{tabular}

\section{Experimental results and discussion}

Taking into account the results of theoretical modeling, the existing approaches to the synthesis of corresponding gold nanostructures were analyzed, which can provide the necessary geometric parameters of nanostructures and the possibility of their LSPR spectrum tuning. It was found that the fabrication protocol for solid gold nanoparticles based on the kinetically-controlled growth from seeds with citrate stabilization [35] allows obtaining colloidal solutions of gold nanoparticles with a diameter from 10 to $\sim 200 \mathrm{~nm}$, which provides the possibility to change the LSPR position from 520 to $720 \mathrm{~nm}$. Methods for synthesis of gold nanoshells on quartz cores $[36,37]$ yield gold shell thickness of 5 to $30 \mathrm{~nm}$ on quartz nanoparticles with a diameter of $50 \ldots 500 \mathrm{~nm}$, providing LSPR position tuning from visible to mid-infrared spectral region. However, these protocols are difficult to implement, costly with respect to chemicals and require multistep chemical synthesis. From this point of view, HGN attract particular interest as the basis for building $\mathrm{SN}$, because their synthesis methods also allow LSPR position tuning in a sufficiently wide range [38]. An additional feature of HGN is a possibility to use cavities inside nanostructures to increase the amount of loaded drug per single nanostructure, which is important to reduce the burden on the patient body that is caused by nanocarriers after drug release.

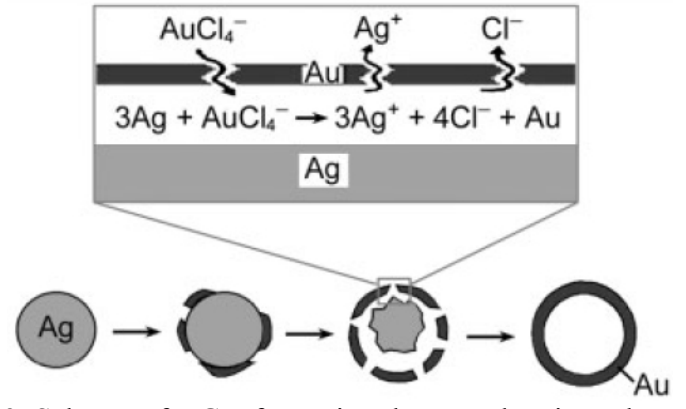

Fig. 2. Scheme of HGN formation due to galvanic replacement reaction of silver with gold [41].

As a result of analysis of hollow metal nanostructures fabrication methods, it was found that one of the most affordable approaches for widespread use, which also yields nanoparticles in relatively large quantities, is the synthesis of hollow nanoparticles on metal nanotemplates using the galvanic replacement reaction [39-42] (Fig. 2). Therefore, in this paper for the synthesis of HGN we adapted the method proposed by Prevo et al. [42] based on galvanic replacement reaction of silver with gold using solid silver nanoparticles in colloidal solution as templates.

The used approach to HGN production consisted of 3 stages: synthesis of seed silver nanoparticles, increasing their size by growth and galvanic replacement reaction of silver with gold using the nanoparticle templates produced in the second stage. In the first stage, seed silver nanoparticles were produced by reduction of $0.2 \mathrm{mM}$ aqueous solution of silver nitrate $(100 \mathrm{ml})$ while adding $100 \mathrm{mM}$ sodium borohydride solution $(2 \mathrm{ml})$ in the presence of $0.5 \mathrm{mM}$ sodium citrate as a stabilizer. The reaction took place at a constant temperature of $60{ }^{\circ} \mathrm{C}$ and stirring using a magnetic stirrer for at least 2 hours. Resulting mixture acquired a yellow color, indicating formation of small and mostly spherical silver nanoparticles. Microphotograph of obtained seed silver nanoparticles from transmission electron microscope (TEM), presented in Fig. 3a, shows a close to spherical shape of nanoparticles whose diameter is $6 \ldots 14 \mathrm{~nm}$.

The second stage of synthesis, growth of silver nanoparticles, was performed after cooling seed silver nanoparticles solution to room temperature. Subsequently, $200 \mathrm{mM}$ aqueous solution of hydroxylamine hydrochloride $(2 \mathrm{~mL})$ was added to the seed solution being mixed. After 5 minutes, the necessary volume of $0.1 \mathrm{M}$ aqueous solution of silver nitrate was added to the resulting solution to increase the final concentration of silver in the solution. In this way, it was possible to adjust the size of silver nanoparticles the more the final concentration of silver in solution was, the larger silver nanoparticles were obtained. Stirring the solution continued for at least 2 hours, leading to the gradual saturation of its yellow color. Microphotograph of the obtained silver nanoparticles with the final concentration of silver in a solution of $0.5 \mathrm{mM}$ is shown 
in Fig. 3b. The shape of obtained silver nanoparticles is mainly spherical; their diameter is $13 \ldots 40 \mathrm{~nm}$. One should note broadening the nanoparticles size spread after the growth process - this issue needs further search for solutions. The light extinction spectra of colloidal solutions of seed and grown silver nanoparticles are shown in Fig. 3c. They exhibit clearly distinguished peaks near the wavelengths of 392 and $403 \mathrm{~nm}$ that are attributed to the LSPR excitation on smaller and larger silver nanoparticles, respectively.
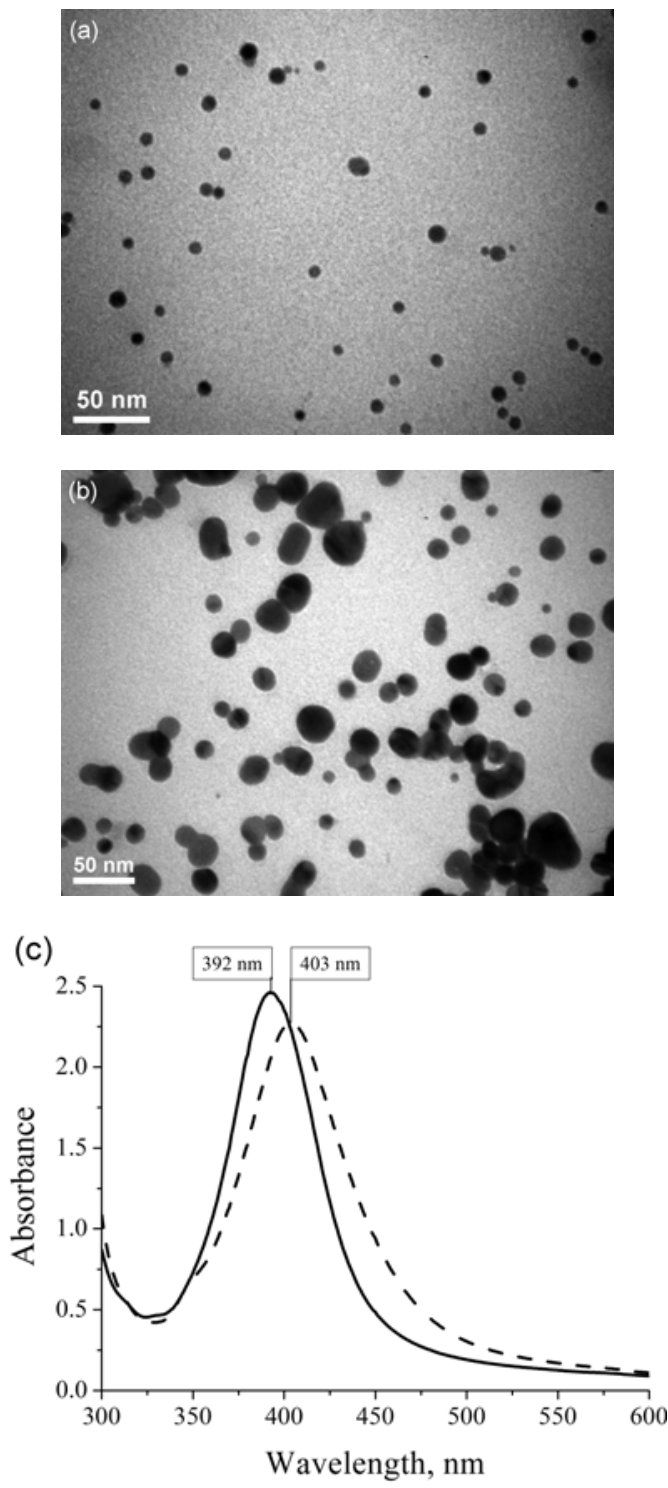

Fig. 3. (a) TEM microphotograph of seed silver nanoparticles obtained by reduction of $0.2 \mathrm{mM}$ silver nitrate solution. (b) TEM microphotograph of silver nanoparticles obtained by growth of seed silver nanoparticles to a final silver concentration of $0.5 \mathrm{mM}$. (c) The measured light extinction spectra of colloidal solutions of seed silver nanoparticles with the silver concentration in solution of $0.2 \mathrm{mM}$ (solid line) and grown silver nanoparticles with the silver concentration in solution of $0.5 \mathrm{mM}$ (dashed line).
At the third stage of synthesis, the obtained colloidal solutions of silver nanoparticles participated in the galvanic replacement reaction of silver with gold. In this process, solution of the grown silver nanoparticles was heated to a constant temperature of $60^{\circ} \mathrm{C}$ while stirring. Then, a certain amount of $25 \mathrm{mM}$ aqueous solution of chloroauric acid was added gradually to the reaction mixture on the basis that the molar ratio $\mathrm{Au}: \mathrm{Ag}$ in the solution should reach 1:3 (according to the scheme in Fig. 2). For several tens of seconds, color of the solution changed from yellow to violet-blue, following the galvanic replacement reaction with gradual dissolution of silver nanoparticles and formation of gold film on their surface. After a stable color was reached, the additional amount of $25 \mathrm{mM}$ aqueous solution of chloroauric acid was added gradually to achieve a complete disappearance of the short-wave LSPR peak of silver nanoparticles, which was monitored by means of spectrophotometric measurements of its intensity in the light extinction spectrum.

Microphotograph of HGN prepared from colloidal solution of silver nanoparticles with the silver concentration of $0.5 \mathrm{mM}$ used as templates is shown in Fig. 4a. The shape of obtained gold nanoparticles is mainly spherical; their geometric sizes are $17 \ldots 42 \mathrm{~nm}$ (in diameter) and 6..12 $\mathrm{nm}$ (wall thickness). The bright areas on the image correspond to cavities and, probably, pores in the HGN walls, and the dark areas are attributed to HGN walls and solid nanoparticles. It should be noted that the resulting geometric parameters of HGN are consistent with the geometric parameters of output templates, silver nanoparticles; no significant increase in the width of their size spread was observed. The measured light extinction spectrum of HGN colloidal solution derived from colloidal silver nanoparticles solution with the silver concentration of $0.5 \mathrm{mM}$ is presented in Fig. $4 \mathrm{~b}$. The resulting spectrum exhibits maximum position equal to $643 \mathrm{~nm}$ that corresponds to the LSPR excitation on hollow gold nanoshells, which is very close to the laser wavelength of $650 \mathrm{~nm}$. It should be noted that the resulting broad LSPR band in the HGN light extinction spectrum ensures PPTE conditions for colloidal solutions of nanostructures, when being irradiated with laser light of different wavelengths.

To study the possibility of LSPR spectrum tuning for HGN, light extinction spectrum changes were registered for colloidal solutions of silver nanoparticles with the silver concentration of $0.5 \mathrm{mM}$ during the galvanic replacement reaction (Fig. 5). Changing the volume of the added chloroauric acid solution enables different values of LSPR position in the light extinction spectra of fabricated HGN to be obtained, which is due to varying wall thickness of the nanostructures. It should be noted that the rate of chloroauric acid solution addition (gradually or the whole volume at once) also affects the final HGN LSPR wavelength (spectra not shown). Specifically, increasing the rate of solution addition led to the long-wave shift and broadening the LSPR band in the HGN light extinction spectrum. 


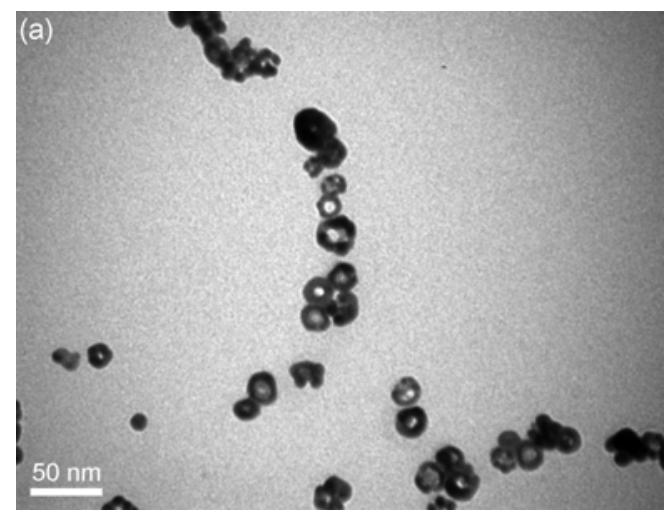

(b)

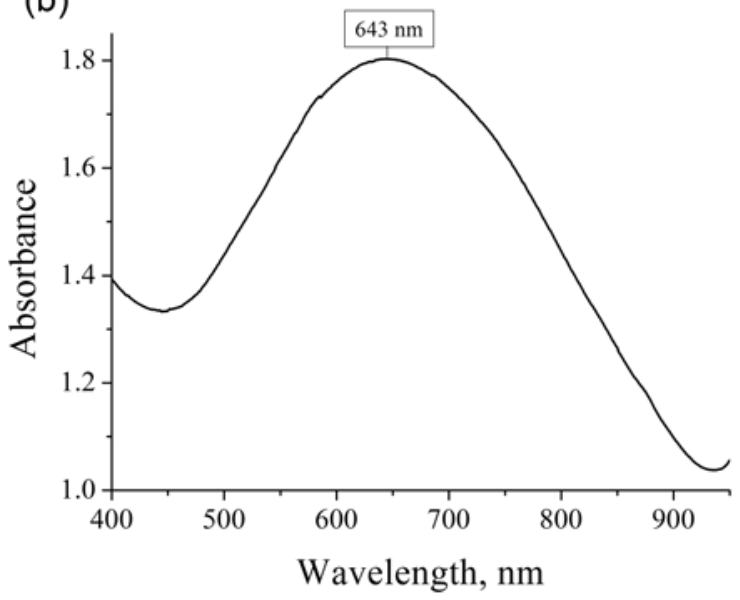

Fig. 4. (a) TEM microphotograph and (b) the measured light extinction spectrum of colloidal HGN solution derived from colloidal silver nanoparticles solution with the silver concentration of $0.5 \mathrm{mM}$ as templates.

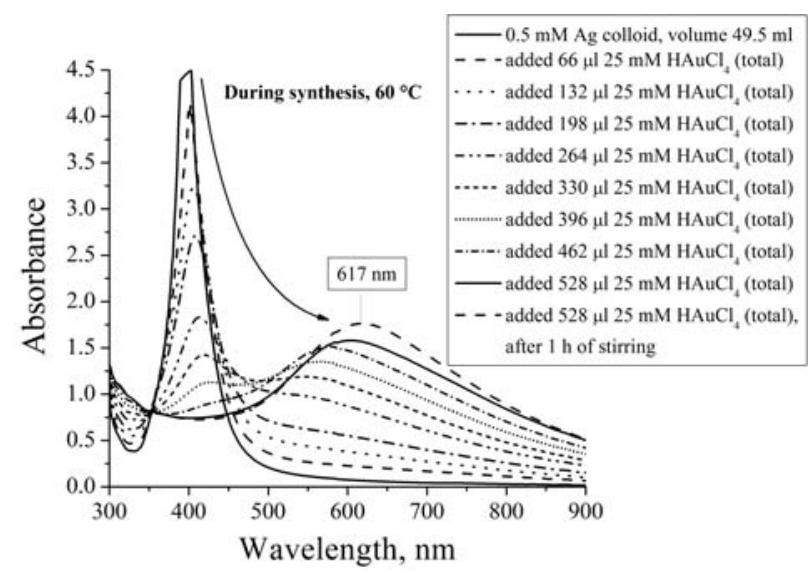

Fig. 5. Measured light extinction spectra for colloidal solution of silver nanoparticles with the silver concentration of $0.5 \mathrm{mM}$ during the galvanic replacement reaction of silver with gold when gradually increasing the volume of added $25 \mathrm{mM}$ solution of chloroauric acid.

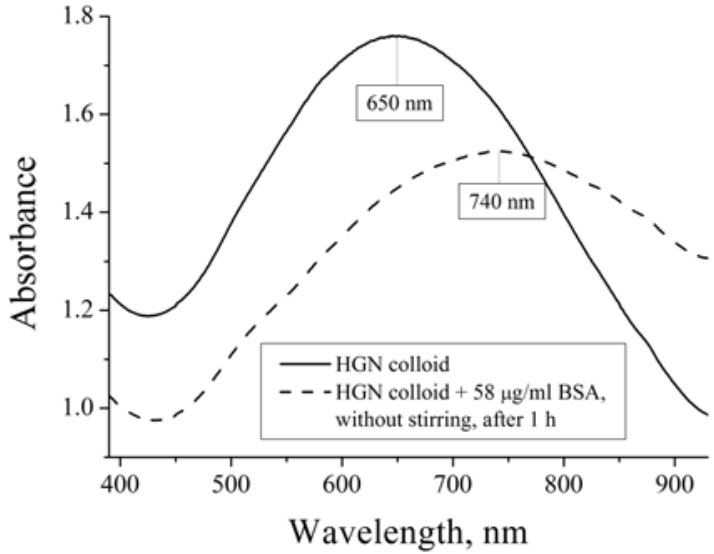

Fig. 6. Measured light extinction spectra of colloidal HGN solution before and after 1 hour since adding BSA in a final concentration of $58 \mu \mathrm{g} / \mathrm{ml}$ without stirring.

To simulate the process of loading drugs into the developed nanocarrier based on HGN, changes in the light extinction spectrum of colloidal HGN solution caused by adsorption of biomolecules onto the external and internal surfaces of HGN were investigated using a model molecule, bovine serum albumin (BSA). Fig. 6 shows the measured light extinction spectra of colloidal HGN solution before and after 1 hour since adding BSA in a final concentration of $58 \mu \mathrm{g} / \mathrm{ml}$ without intensive stirring of the solution. In such a way, the natural diffusion of biomolecules with the possibility of gradual penetration into the internal cavities of HGN was provided. As a result, there was a significant long-wave shift of the LSPR peak in the light extinction spectrum, which reached $90 \mathrm{~nm}$ and led to the expected shift of HGN LSPR position to the near infrared region. This result indicates that taking into account changes in the SN LSPR spectrum, which occur when loading drugs, is necessary to match the SN LSPR position with a laser wavelength that should induce the drug release. In addition, significant sensitivity of LSPR spectrum to adsorption of biomolecules can be used to develop a HGN-based biosensor, which will be able to detect biomolecular analytes in much lower concentrations, as compared to biosensors based on solid gold nanoparticles $[43,44]$ that exhibit less pronounced LSPR peak shifts for the same analyte at comparable or higher concentrations.

\section{Conclusion}

Using the Mie theory for a spherical particle with a homogeneous shell, theoretical study of light extinction spectral dependences was performed for gold nanostructures of different morphology that can support LSPR excitation in the red and near infrared spectral regions and act as a basis for building smart nanocarriers for drug delivery based on plasmon-enhanced 
photothermal effect upon the laser irradiation. It was found that LSPR excitation at the wavelengths 650 and $808 \mathrm{~nm}$, which correspond to the standard laser light sources, can be provided by solid gold nanoparticles with a diameter of about 150 and $210 \mathrm{~nm}$, respectively, and gold nanoshells with a thickness of about 1 to $8 \mathrm{~nm}$ on dielectric cores with a diameter of $20 \ldots 100 \mathrm{~nm}$. The analysis of protocols for producing these nanostructures has shown that the most affordable, quick and easy method for building a smart nanocarrier of this type is the synthesis of hollow gold nanostructures on nanotemplates using the galvanic replacement reaction. The method for fabrication of hollow gold nanoshells on the basis of this synthesis was implemented, which supports changing the geometric parameters of nanostructures and, therefore, tuning their LSPR position. Spectral and morphological properties of the obtained nanostructures were analyzed at each stage of their preparation. It was demonstrated that loading model biomolecules into hollow gold nanoshells leads to a significant red shift of LSPR position and, therefore, requires additional matching with the laser wavelength for maximum plasmon-enhanced photothermal effect that should be taken into account when developing smart nanocarriers. Mathematical modeling these multicomponent systems will be a topic for future research.

\section{Acknowledgements}

This work was supported by the Science and Technology Center in Ukraine (project 6044 for 2015 to 2017).

\section{References}

1. K.K. Jain, Future of nanomedicine: impact on healthcare \& society // Nanomedicine, 10(21), p. 3199-3202 (2015).

2. M.S. Singh and D. Peer, RNA nanomedicines: the next generation drugs? // Curr. Opin. Biotechnol. 39, p. 28-34 (2016).

3. C.M. Castro, H. Im, H. Lee and R. Weissleder, Nanotechnology approaches for intraprocedural molecular diagnostics, Chap. 12, in: Imaging and Visualization in The Modern Operating Room, Eds. Y. Fong, P.C. Giulianotti, J. Lewis, B.G. Koerkamp, T. Reiner. Springer, New York, 2015, p. 157-166.

4. I. Khan, M. Khan, M.N. Umar and D.H. Oh, Nanobiotechnology and its applications in drug delivery system: a review // IET Nanobiotechnol. 9(6), p. 396-400 (2015).

5. N. Habibi, N. Kamaly, A. Memic and H. Shafiee, Self-assembled peptide-based nanostructures: smart nanomaterials toward targeted drug delivery // Nano Today, 11(1), p. 41-60 (2016).

6. K. Yang, L. Feng and Z. Liu, The advancing uses of nano-graphene in drug delivery // Expert Opin. Drug Deliv. 12(4), p. 601-612 (2015).
7. H.A. Santos, L.M. Bimbo, L. Peltonen and J. Hirvonen, Inorganic nanoparticles in targeted drug delivery and imaging, Chap. 18, in: Targeted Drug Delivery: Concepts and Design. Eds. P.V. Devarajan, S. Jain. Springer Intern. Publ. 2015, p. 571-613.

8. H. Daraee, A. Eatemadi, E. Abbasi, S. Fekri Aval, M. Kouhi and A. Akbarzadeh, Application of gold nanoparticles in biomedical and drug delivery // Artif. Cells Nanomed. Biotechnol. 44(1), p. 410422 (2016).

9. G.F. Paciotti, J. Zhao, S. Cao, P.J. Brodie, L. Tamarkin, M. Huhta, L.D. Myer, J. Friedman and D.G.I. Kingston, Synthesis and evaluation of paclitaxel-loaded gold nanoparticles for tumortargeted drug delivery // Bioconjugate Chem. Article ASAP (2016).

10. M. Hembury, C. Chiappini, S. Bertazzo et al., Gold-silica quantum rattles for multimodal imaging and therapy // Proc. Natl. Acad. Sci. USA, 112(7), p. 1959-1964 (2015).

11. B. Duncan, C. Kim and V.M. Rotello, Gold nanoparticle platforms as drug and biomacromolecule delivery systems // J. Control. Release, 148(1), p. 122-127 (2010).

12. S. Rana, A. Bajaj, R. Mout and V.M. Rotello, Monolayer coated gold nanoparticles for delivery applications // Adv. Drug Deliv. Rev. 64(2), p. 200216 (2012).

13. X.Q. Zhao, T.X. Wang, W. Liu, C.D. Wang, D. Wang, T. Shang, L.H. Shen and L. Ren, Multifunctional Au@IPN-pNIPAAm nanogels for cancer cell imaging and combined chemophotothermal treatment // J. Mater. Chem. 21(20), p. 7240-7247 (2011).

14. T. Kawano, Y. Niidome, T. Mori, Y. Katayama and T. Niidome, PNIPAM gel-coated gold nanorods for targeted delivery responding to a near-infrared laser // Bioconjugate Chem. 20(2), p. 209-212 (2009).

15. A.M. Elliott, R.J. Stafford, J. Schwartz, J. Wang, A.M. Shetty, C. Bourgoyne, P. O’Neal and J.D. Hazle, Laser-induced thermal response and characterization of nanoparticles for cancer treatment using magnetic resonance thermal imaging // Med. Phys. 34(7), p. 3102-3108 (2007).

16. J.M. Stern, J. Stanfield, W. Kabbani, J.T. Hsieh and J.A. Cadeddu, Selective prostate cancer thermal ablation with laser activated gold nanoshells // J. Urol. 179(2), p. 748-753 (2008).

17. I.H. El-Sayed, X. Huang and M.A. El-Sayed, Selective laser photo-thermal therapy of epithelial carcinoma using anti-EGFR antibody conjugated gold nanoparticles // Cancer Lett. 239(1), p. 129135 (2006).

18. Z. Liang, X. Li, Y. Xie and S. Liu, 'Smart' gold nanoshells for combined cancer chemotherapy and hyperthermia // Biomed. Mater. 9(2), 025012 (2014). 
19. V. Voliani, G. Signore, R. Nifosí, F. Ricci, S. Luin and F. Beltram, Smart delivery and controlled drug release with gold nanoparticles: new frontiers in nanomedicine // Recent Pat. Nanomed. 2(1), p. 3444 (2012).

20. A.M. Smith, M.C. Mancini and S. Nie, Second window for in vivo imaging // Nat. Nanotechnol. 4(11), p. 710-711 (2009).

21. W.A. Murray, B. Auguié and W.L. Barnes, Sensitivity of localized surface plasmon resonances to bulk and local changes in the optical environment // J. Phys. Chem. C, 113(13), p. 51205125 (2009).

22. H.N. Xie, I.A. Larmour, Y.C. Chen, A.W. Wark, V. Tileli, D.W. McComb, K. Faulds and D. Graham, Synthesis and NIR optical properties of hollow gold nanospheres with LSPR greater than one micrometer // Nanoscale, 5(2), p. 765-771 (2013).

23. N. Halas, Playing with plasmons: tuning the optical resonant properties of metallic nanoshells // MRS Bull. 30(5), p. 362-367 (2005).

24. A.M. Alkilany, L.B. Thompson, S.P. Boulos, P.N. Sisco and C.J. Murphy, Gold nanorods: their potential for photothermal therapeutics and drug delivery, tempered by the complexity of their biological interactions // Adv. Drug Deliv. Rev. 64(2), p. 190-199 (2012).

25. S.E. Skrabalak, L. Au, X. Li and Y. Xia, Facile synthesis of Ag nanocubes and Au nanocages // Nat. Protoc. 2(9), p. 2182-2190 (2007).

26. Y. Jin and X. Gao, Spectrally tunable leakage-free gold nanocontainers // J. Am. Chem. Soc. 131(49), p. 17774-17776 (2009).

27. A.M. Lopatynskyi, O.G. Lopatynska, L.J. Guo and V.I. Chegel, Localized surface plasmon resonance biosensor - Part I: theoretical study of sensitivity extended Mie approach // IEEE Sens. J. 11(2), p. 361-369 (2011)

28. W. Lv, P.E. Phelan, R. Swaminathan, T.P. Otanicar and R.A. Taylor, Multifunctional core-shell nanoparticle suspensions for efficient absorption // J. Sol. Energy Eng. 135(2), 021004 (2013).

29. P.B. Johnson and R.W. Christy, Optical constants of the noble metals // Phys. Rev. B, 6(12), p. 43704379 (1972).

30. L.I. Berger, Fermi energy and related properties of metals, in: Handbook of Chemistry and Physics, 84-th ed. Ed. R.D. Lide. CRC Press, Boca Raton, FL, 2004, p. 12-232-12-233.

31. A.I. Gusev and A.A. Rempel, Nanocrystalline Materials. Nauka, Moscow, Russian Federation, 2001 (in Russian).
32. U. Kreibig and M. Vollmer, Optical Properties of Metal Clusters (Springer Series in Materials Science, vol. 25). Springer, Berlin, Germany, 1995.

33. N.L. Dmitruk, A.V. Goncharenko and E.F. Venger, Optics of Small Particles and Composite Media. Naukova dumka, Kyiv, 2009 (in Ukrainian).

34. C.G. Granqvist and O. Hunderi, Optical absorption of ultrafine metal spheres with dielectric cores // Z. Physik, 30(1), p. 47-51 (1978).

35. N.G. Bastús, J. Comenge and V. Puntes, Kinetically controlled seeded growth synthesis of citrate-stabilized gold nanoparticles of up to 200 $\mathrm{nm}$ : size focusing versus Ostwald ripening // Langmuir, 27(17), p. 11098-11105 (2011).

36. L.R. Hirsch, A.M. Gobin, A.R. Lowery, F. Tam, R.A. Drezek, N.J. Halas and J.L. West, Metal nanoshells // Ann. Biomed. Eng. 34(1), p. 15-22 (2006).

37. S.J. Oldenburg, R.D. Averitt, S.L. Westcott and N.J. Halas, Nanoengineering of optical resonances // Chem. Phys. Lett. 288(2-4), p. 243-247 (1998).

38. J.Z. Zhang, Biomedical applications of shapecontrolled plasmonic nanostructures: a case study of hollow gold nanospheres for photothermal ablation therapy of cancer // J. Phys. Chem. Lett. 1(4), p. 686-695 (2010).

39. Y. Sun, B.T. Mayers and Y. Xia, Templateengaged replacement reaction: a one-step approach to the large-scale synthesis of metal nanostructures with hollow interiors // Nano Lett. 2(5), p. 481-485 (2002).

40. E. Hao, S. Li, R.C. Bailey, S. Zou, G.C. Schatz and J.T. Hupp, Optical properties of metal nanoshells // J. Phys. Chem. B, 108(4), p. 1224-1229 (2004).

41. Y. Sun, B. Mayers and Y. Xia, Metal nanostructures with hollow interiors // Adv. Mater. 15(7-8), p. 641-646 (2003).

42. B.G. Prevo, S.A. Esakoff, A. Mikhailovsky and J.A. Zasadzinski, Scalable routes to gold nanoshells with tunable sizes and response to near-infrared pulsed-laser irradiation // Small, 4(8), p. 1183-1195 (2008).

43. L. Olofsson, T. Rindzevicius, I. Pfeiffer, M. Käll and Fredrik Höök, Surface-based gold-nanoparticle sensor for specific and quantitative DNA hybridization detection // Langmuir, 19(24), p. 10414-10419 (2003).

44. K. Fujiwara, H. Watarai, H. Itoh, E. Nakahama and N. Ogawa, Measurement of antibody binding to protein immobilized on gold nanoparticles by localized surface plasmon spectroscopy // Anal. Bioanal. Chem. 386, p. 639-644 (2006). 